\title{
1 TaxisPy: A Python-based Software for the Quantitative Analysis of 2 Bacterial Chemotaxis
}

3

4

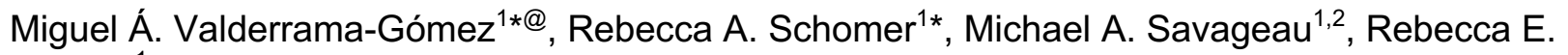 \\ Parales ${ }^{1}$ \\ ${ }^{1}$ Department of Microbiology \& Molecular Genetics, College of Biological Sciences, University of \\ California, Davis, USA \\ ${ }^{2}$ Department of Biomedical Engineering, University of California, Davis, USA \\ ${ }^{*}$ Authors equally contributed to this work \\ @ Corresponding Author: mavalderramagomez@ucdavis.edu
}

\begin{abstract}
Several species of bacteria are able to modify their swimming behavior in response to chemical attractants or repellents. Methods for the quantitative analysis of bacterial chemotaxis such as quantitative capillary assays are tedious and time-consuming. Computer-based video analysis of swimming bacteria represents a valuable method to directly assess their chemotactic response. Even though multiple studies have used this approach to elucidate various aspects of the bacterial chemotaxis, to date, no computer software for such analyses is freely available. Here, we introduce TaxisPy, a Python-based software for the quantitative analysis of bacterial chemotaxis. The software comes with an intuitive graphical user interface and can be easily accessed through Docker on any operating system. Using a video of freely swimming cells as input, TaxisPy estimates the culture's average tumbling frequency over time. We demonstrate the utility of the software by assessing the effect of different concentrations of the attractant shikimate on the swimming behavior of Pseudomonas putida F1 and by capturing the adaptation process that Escherichia coli undergoes after being exposed to L-aspartate.
\end{abstract}

\section{Keywords}

Quantitative chemotaxis, video analysis software, cell tracking software. 


\section{Introduction}

Environmental conditions such as temperature, light intensity and chemical composition influence the ability of an organism to grow, reproduce and survive. Many bacteria have evolved intricate mechanisms to sense these and other external stimuli. These signals are processed by the cell and directly affect the functioning of its motility apparatus, thus leading to an informed displacement towards a beneficial environment or away from unfavorable conditions.

Chemotaxis refers to a modification in the motility pattern of an organism in response to a change in the chemical composition of its environment. Flagellated bacteria such as Escherichia coli and Pseudomonas putida swim in liquid environments by rotating their helical flagella. A change in the swimming direction is achieved by the intermittent change of the direction of flagellar rotation, which leads to tumbles that usually last only a fraction of a second (Webre et al. 2003). The swimming pattern of a cell can be quantitatively characterized by its tumbling frequency (Alon et al. 1999, Pohl et al. 2017). In the absence of chemical attractants, the swimming pattern of motile bacteria is described as a "random walk", which is characterized by short periods of smooth swimming (often termed "runs") and a high tumbling frequency, with typical values of 0.44 changes of direction per second for E. coli (Alon et al. 1999, Staropoli and Alon 2000). After a chemical attractant is sensed, chemotactic bacteria decrease their tumbling frequency to values lower than 0.05 per second (Alon et al. 1999). As a result, cells in a culture effectively swim towards higher concentrations of the attractant following straight paths.

Over the last decades, qualitative and quantitative methods for the study of bacterial chemotaxis have been developed. Some representative protocols include capillary assays (Pfeffer 1884, Adler 1969, Adler 1973), chemical-in-plug assays (Tso and Adler 1974) and computer-based video analyses of swimming cells (Berg and Brown 1972). See Ditty and Parales 2015 for an overview of these methods. While qualitative methods are fast and usually do not involve major technical difficulties, quantitative methods such as the quantitative capillary assay are tedious and time-consuming. Computer-based video analysis of swimming bacteria represents a valuable method to directly and quantitatively assess the chemotactic response of bacteria. Even though multiple studies have used this approach to elucidate various aspects of bacterial chemotaxis (Harwood et al. 1989, Alon et al. 1998, Alon et al. 1999, Staropoli and Alon 2000), to date, no computer software for such analyses is freely available. Here, we introduce TaxisPy, a Python-based software for the quantitative analysis of bacterial chemotaxis. The software comes with an intuitive graphical user interface that is especially suited for users with limited programming knowledge. It systematically addresses typical difficulties associated with 
the customized use of cell tracking software such as filtering of atypical cell trajectories and determination of parameter values for the identification of tumbles. TaxisPy can be easily accessed through Docker on any operating system.

\section{Materials and Methods}

\subsection{Bacterial strains, media composition and attractants}

The responses of E. coli K12 strain RP437 [thr leu his metF eda rpsL thi ara lacY xyl tonA tsx] (Parkinson 1978) and Pseudomonas putida F1 -both motile organisms used as models for studying chemotactic behavior- to chemical attractants were examined in this study. All cells were grown in Luria-Bertani medium (LB; Sambrook et al. 1989) or minimal medium (MSB; Stanier et al. 1996) at $30^{\circ} \mathrm{C}$ in a shaking incubator.

\subsection{Behavioral assays}

Pseudomonas putida cultures were grown for approximately 18 hours in minimal MSB medium containing $10 \mathrm{mM}$ succinate at $30^{\circ} \mathrm{C}$. One hundred microliters of the culture were subcultured into $15 \mathrm{~mL}$ minimal MSB containing $10 \mathrm{mM}$ succinate and $5 \mathrm{mM}$ shikimate for induction of chemotactic behavior towards aromatic acids (Luu 2015). After approximately 6 hours of growth, the cells were harvested at mid-exponential phase $\left(\mathrm{OD}_{600}=0.4-0.6\right)$ by centrifugation at $5,000 \mathrm{rpm}$ for 10 minutes at room temperature. The cells were gently washed in $15 \mathrm{ml}$ of chemotaxis buffer (CB; 50 mM potassium phosphate buffer $\mathrm{pH}$ 7.0, $10 \mu \mathrm{M}$ disodium EDTA, $0.05 \%$ glycerol) and re-centrifuged for 10 minutes at 5,000 rpm (Parales et al. 2000). Finally, the cells were resuspended in $15 \mathrm{~mL}$ of aerated CB. For analysis of the swimming pattern of $P$. putida, $10 \mu$ of the cell suspension was placed on a glass slide (Fisher Scientific) and mixed with $1 \mu \mathrm{l}$ of various concentrations of shikimate in $\mathrm{CB}$ to attain final concentrations of $0 \mu \mathrm{M}, 10$ $\mu \mathrm{M}, 50 \mu \mathrm{M}, 100 \mu \mathrm{M}$, and $1 \mathrm{mM}$. Two seconds after the addition of the attractant, video recording was initiated. Recording of the samples was performed with an Infinity Lite microscope camera (Lumenera, Ottawa, ON, Canada) mounted to a Nikon eclipse TE2000-S inverted microscope, using a magnification of 400X. The swimming behavior was recorded for 1 minute at a speed of 20.3 frames per second using the software Infinity Capture 6.5.4. These videos were then analyzed with TaxisPy as described below to estimate the tumbling frequency of the cell populations. 
Samples for analyzing the adaptation of $E$. coli after stimulation with L-aspartate were generated using a different set-up. Cells were grown overnight in LB medium before being

123 harvested by centrifugation (10,000 RPM, 1 minute) and washed with an equal volume of MSB

124 medium. Cells were resuspended in an equal volume of MSB medium. Two hundred microliters

125 of washed culture were inoculated into $15 \mathrm{~mL}$ of minimal MSB medium containing $12 \mathrm{mM}$

126 glucose. When the cultures reached mid-exponential phase $\left(O D_{600}=0.4-0.6\right), 1 \mathrm{~mL}$ aliquots of

127 culture were harvested by centrifugation at 5,000 rpm for $10 \mathrm{~min}$ at room temperature and

128 gently resuspended in $1 \mathrm{~mL}$ of $\mathrm{CB}$. At regular time intervals, $10 \mu \mathrm{L}$ samples of $E$. coli were

129 removed and observed with a Nikon eclipse TE200-S inverted microscope at 400X

130 magnification. Using the Infinity Lite mounted camera, 10 second videos were recorded at a

131 speed of 20.3 frames per second. The chemotactic response was initiated by adding L-

132 aspartate to the resuspended cultures at final concentration of $1 \mathrm{mM}$. To keep cells motile

133 during the experiment, cells were incubated at $30^{\circ} \mathrm{C}$ in a shaking incubator (200 rpm). These

134 videos were then analyzed with TaxisPy as described below to estimate the tumbling

135 frequencies of the cell populations.

136

137

138

139

140

141

142

143

144

145

146

147

148

149

150

151

152

153

154

\subsection{Software and General Workflow}

TaxisPy integrates several Python packages to enable the estimation of the tumbling frequency of a bacterial culture (Fig. 1). Its intuitive graphical user interface is based on Jupyter widgets and contains six different tabs (Fig. 2). In order to streamline its distribution, especially among users with limited programming knowledge, TaxisPy can be accessed via a Docker image. Docker is a computer program that performs operating-system-level virtualization and is used to run software packages called containers. Containers bundle their own application, tools, libraries and configuration files and are created from images. Docker has been increasingly used to distribute software across different operating systems in a fast and secure way. To download TaxisPy via Docker, four steps are required:

1. Install Docker on your computer. Docker is supported by all major operating systems: Linux, MacOs and Windows.

2. Download the latest TaxisPy image by typing the following command on a Terminal (Mac, Linux) or Command Prompt window (Microsoft Windows): 
3. Start a Docker container to access TaxisPy by typing the following command on the same window:

docker run $-\mathrm{d}-\mathrm{p} 8888: 8888 \mathrm{mlvg} /$ taxispy

That command will create a container without access to the files of the host computer (i.e., your computer). Files created within the container will be lost after the container is stopped. In order to grant access to files on the host computer, the previous command should be complemented with the flag --mount:

docker run $-\mathrm{d}-\mathrm{p}$ 8888:8888 --mount

type=bind, source=/Users, target=/Documents/host mlvg/taxispy

Windows users should use:

docker run -d -p 8888:8888 --mount

type=bind, source $=/ /$ c/Users, target=/Documents / host

$$
\text { m1vg/taxispy }
$$

4. Access TaxisPy by opening the following address on any internet browser (e.g., Google Chrome):

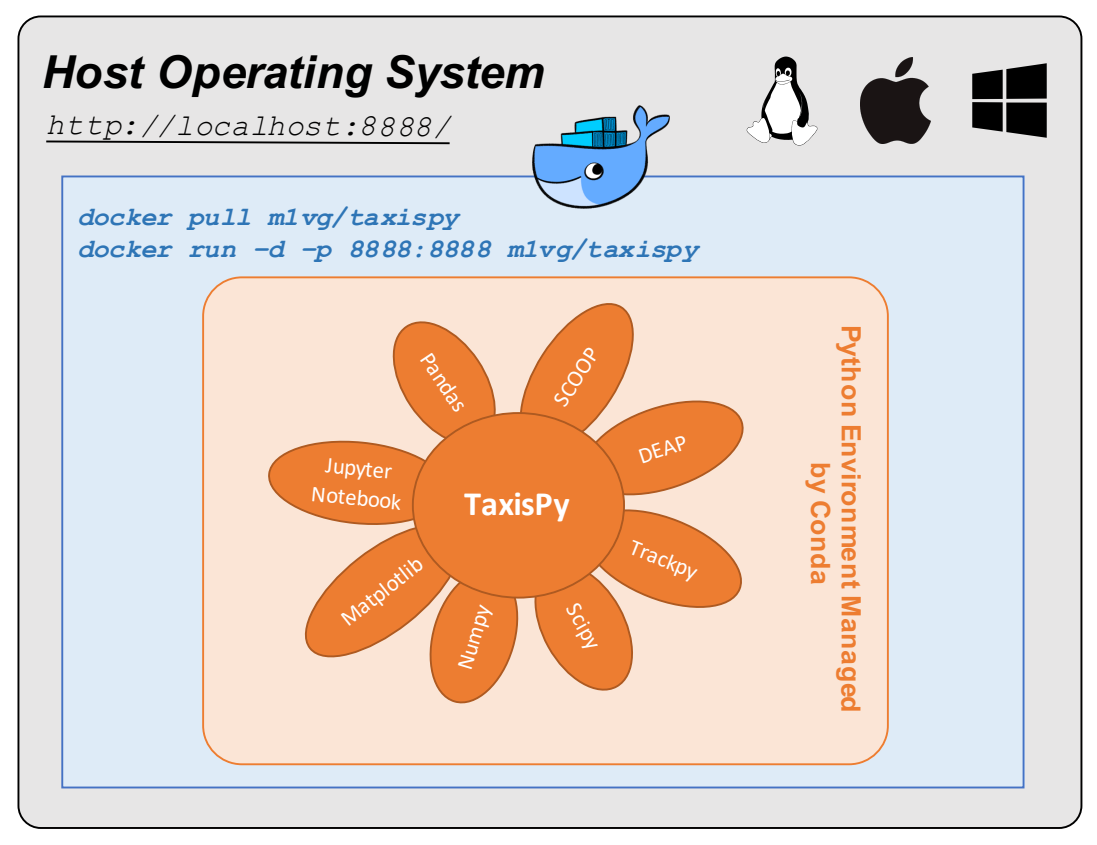

Figure 1. Software architecture. TaxisPy bundles several Python packages that together allow a computer-assisted, quantitative analysis of bacterial chemotaxis. TaxisPy's user interface is based on Jupyter widgets and can be accessed from Jupyter Notebooks on any web browser, e.g., Google Chrome. TaxisPy's ability to identify cells and generate trajectories is enabled by the Python package Trackpy (Allan et al. 2019). Pandas, Numpy and Scipy are additional packages that provide TaxisPy with computational 179 objects and functions required to store, filter and process the data generated by Trackpy. The python 
packages DEAP (Fortin et al. 2012) and Scoop (Hold-Geoffroy et al. 2014) are used to estimate parameter values for the identification of tumbles. All plotting routines are supported by Matplotlib.

Quantitative characterization of the swimming behavior of a given bacterial culture by means of its tumbling frequency involves five consecutive steps, as shown in Fig. 2. First, a video registering freely swimming cells is split into its constituting frames. Each frame represents a snapshot of the culture at a given time point. Splitting can be done by the software FFmpeg (it supports a wide range of video formats), for which a simple user interface is provided within the TaxisPy Docker image; or by any other software designed for that purpose. Then, cells present in each frame are located and swimming trajectories identified by linking the position of individual cells through subsequent frames. Within TaxisPy, this functionality is provided by Trackpy 0.4.2 (Allan et al. 2019), a Python package that implements feature-finding and linking algorithms originally introduced by Crocker and Grier (1996). The next step consists of calculating the number of tumbles for each trajectory. Previous reports have used motion properties of individual trajectories - e.g., acceleration and velocities- to calculate the number of tumbles for each cellular trajectory. The underlying idea is that a cell decreases its linear velocity and increases its absolute acceleration below/above certain thresholds while tumbling. Specific threshold values were empirically determined by the authors of these studies (Berg and Brown 1972, Sager et al. 1988, Harwood 1989, Amsler 1996, Alon et al. 1998). It is clear that these values are a function of a variety of biological (i.e., bacterial strain, growth conditions, optical density, etc.) and technical (i.e., frame rate, signal smoothing) factors, which restricts the utility of such thresholds to the specific conditions for which they were estimated. TaxisPy addresses this issue by using a condition-specific training set consisting of a set of cellular trajectories with known changes of direction, along with a genetic algorithm. The generation of this training set by the user is supported by functionalities provided by TaxisPy. To estimate the number of tumbles for a given cellular trajectory, TaxisPy requires values for three different parameters: a threshold value for the absolute acceleration and values for two parameters controlling the smoothing routines: \# Frames and \# Smooth (see Table S1 in the Supplemental Information). Optimal values for these parameters are estimated based on a user-defined training set, so that the squared difference between observed and calculated number of tumbles by TaxisPy is minimized. TaxisPy uses the functionality provided by the Python package DEAP (Fortin et al. 2012) to solve this optimization problem. The final step in the characterization of the swimming behavior of a bacterial culture involves the determination of its average tumbling frequency. This value is calculated by dividing the total number of tumbles estimated for a set of 
215 trajectories over the total duration of those trajectories. Alternatively, the tumbling frequency can

216 be calculated for each individual trajectory and an average over all trajectories can be used to

217 estimate the average tumbling frequency of the culture. TaxisPy offers the flexibility to select the

218 desired calculation method. In this work, reported tumbling frequencies were calculated using

219 the first method, which divides the total number of tumbles over the total duration of all

220 trajectories. In order to exclude anomalous trajectories that might bias the average tumbling

221 frequency calculated for a set of trajectories, TaxisPy offers a series of filters to remove

222 trajectories of nonmotile cells, trajectories exhibiting a too high number of tumbles (e.g., "stuck"

223 cells that are trying to detach) or trajectories with a linear velocity that is abnormally low. Refer

224 to the tutorial contained in the Docker image under /Tutorial for a more detailed description of

225 this workflow.

226

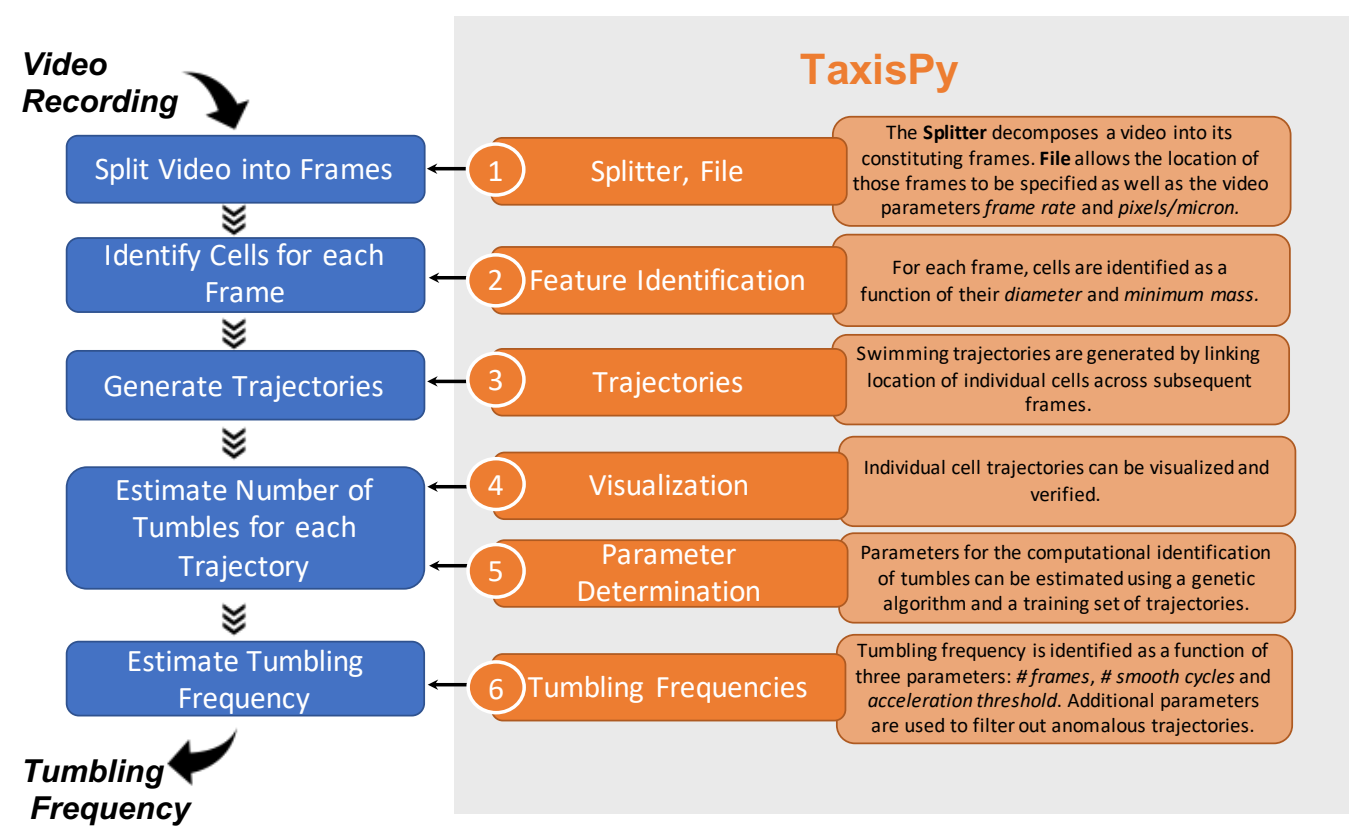

Figure 2. From a video recording to the bacterial tumbling frequency using TaxisPy. Five different tasks -represented by the blue rectangles- are involved in estimating the tumbling frequency of a bacterial culture from a video of its swimming behavior. TaxisPy's user interface consists of six sequentially arranged tabs -represented by the orange rectangles- that provide required functionalities to perform each one of these tasks. Even though several parameters are involved in various steps of the analysis, TaxisPy provides necessary tools to identify appropriate values for those parameters.

\subsection{Key parameter values required by TaxisPy}

A comprehensive list of all parameters required by TaxisPy, along with a short description and nominal values is provided in Table S1 of the Supplemental Information. Eleven key parameters are involved in different stages of the analysis. These include parameters such as 
241 Diameter and Min. Mass, which are used in the Feature Identification tab and are directly

242 passed to Trackpy for the identification of individual cells in each frame. A nominal value of 25

243 pixels for Diameter and 2,000 for Min. Mass were used for all video analyses of both $P$. putida

244 and E. coli cultures. TaxisPy offers an intuitive way to identify optimal values for these two

245 parameters by visually inspecting the ability of the software to correctly identify cells in three

246 different frames, corresponding to the first, middle, and last frame of a given set. Cells identified

247 in these frames are enclosed by a blue circle by TaxisPy. Appropriate values for Diameter and

248 Min. Mass will maximize the number of cells correctly identified by TaxisPy.

A second group of key parameters consists of \# Trajectories, Trajectory \# and \# Chng.

250 Dir. They refer to the number of trajectories within a given user-defined training set, the ID of

251 each trajectory and the number of changes of direction or tumbles for each trajectory,

252 respectively. These parameters define the training set that is used by DEAP to identify optimal

253 values for smoothing parameters (\#Frames and \#Smooth) and a value for the acceleration

254 threshold (Acc. Thrhld.), which are used to identify the number of tumbles of trajectories not

255 contained within the training set. The genetic algorithm implemented by DEAP used a

256 population size of 100 individuals and 5 generations. The initial population was generated at

257 random, using bounds on Acc. Thrhld of 1000 micron $/ \mathrm{s}^{2}$ and \#Frames and \#Smooth of 5. To

258 increase variability within the population, we applied a two-point crossover and gaussian

259 mutation with mean of 0 and standard deviation of 0.2. The independent probability for each

260 attribute of the population to be mutated was 0.2 . This configuration of the genetic algorithm

261 exhibited a good performance for the training sets used in this study. Population size, number of

262 generations and bounds of key parameters can be customized from the tab Parameter

263 Determination. The training set used by DEAP can be easily generated by the user employing

264 the tabs Trajectories and Visualization. Refer to Tables S2 to S6 in the Supplemental

265 Information for training sets used to identify the acceleration threshold and smoothing

266 parameters for the $P$. putida experiments and to Tables S8 to S10 for training sets used for the

267 E. coli experiments. Optimal parameter values resulting from these training sets are

268 summarized in Table S7 for the $P$. putida videos and in Table S11 for the E. coli.

An additional group of key parameters is located within the tab Tumbling Frequencies.

270 These parameters control the way TaxisPy filters out anomalous trajectories and calculates

271 average tumbling frequencies. The parameters Velocity, Dsplcmt, \% and Max. Chng. Dir. are

272 used to sort out short trajectories, trajectories of cells swimming with too low linear velocities

273 and trajectories exhibiting an excessive number of turns, respectively. Nominal values of 4

$274 \mathrm{micron} / \mathrm{s}, 10 \%$, and 10 tumbles were used to analyze all data presented in this study. Finally, 
275 the parameter $\boldsymbol{T}$. Int. (s) is used to specify the time intervals used by TaxisPy to calculate the

276 temporal evolution of the tumbling frequency of the culture. A value of 5 seconds was used for

277 the analysis of the P. putida experiments, while a value of 10 seconds was used for E. coli.

\section{Results}

In order to demonstrate the utility of TaxisPy, the chemotactic response of two different microorganisms after stimulation with chemical attractants was video recorded and analyzed. Using the frames of the recorded videos as input, TaxisPy allowed the quantification of the effect of shikimate on the tumbling frequency of $P$. putida and the visualization of the adaptation process of $E$. coli after exposure to $1 \mathrm{mM} \mathrm{L}$-aspartate. The following results were generated using parameter values listed in Table S7 for $P$. putida F1 and Table S11 for E. coli. Refer to ReadMe Notebooks contained in the Docker image under /Ecoli_Aspartate and

/Pseudomonas_Shikimate to reproduce individual results.

\subsection{Characterization of the chemotactic response of $\boldsymbol{P}$. putida}

P. putida F1 was exposed to four different concentrations of shikimate. Chemotaxis buffer without shikimate was used as the control condition. The response of the culture under each condition was video recorded in triplicate for sixty seconds and TaxisPy was subsequently used to calculate the average tumbling frequency under each condition as described in the Methods section. Fig. 3 summarizes our findings. As expected, the cellular tumbling frequency decreases from its basal value of 0.42 tumbles/second under unstimulated conditions to a low value of 

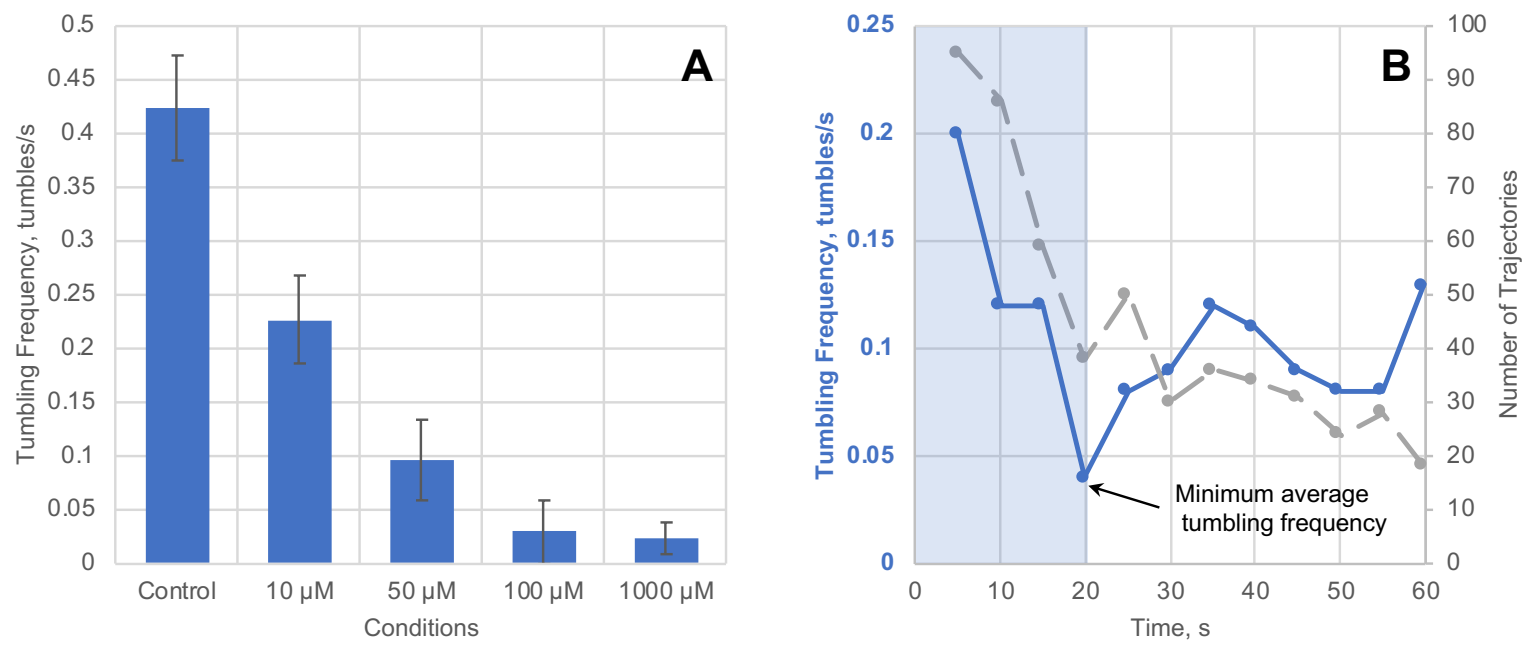

Figure 3. Tumbling frequency of $\boldsymbol{P}$. putida for different shikimate concentrations. A. Tumbling frequency exhibited by the culture as a function of the shikimate concentration. After stimulation with shikimate, the swimming pattern was characterized by the minimum average tumbling frequency exhibited by the cells during the first 20 seconds and calculated using time intervals of 5 seconds. For the control cultures (chemotaxis buffer only), the swimming pattern was characterized by the average tumbling frequency calculated for the first 20 seconds of each video. Refer to main text for details. The number of trajectories used to calculate tumbling frequencies for each video ranged from 257 to 23. Error bars represent one standard deviation of the culture's tumbling frequency calculated from three different videos. B. Temporal evolution of the tumbling frequency of $P$. putida after exposure to $1 \mathrm{mM}$ shikimate is represented by the blue solid line. Average frequencies were calculated using intervals of 5 seconds; the corresponding data point is placed at the end of each interval. The grey dashed line represents the number of cellular trajectories used by TaxisPy to calculate reported average frequencies. The shaded blue area represents the first 20 seconds of the video used to determine the minimum average tumbling frequency.

The minimum tumbling frequency exhibited by the culture within the first 20 seconds of each video was used in Fig. $3 \mathrm{~A}$ to assess the effect of different concentrations of shikimate on the swimming pattern of the culture. This value was used instead of the average tumbling frequency over the same period of time because it better captured the reduction of the culture's tumbling frequency after exposure to the chemical attractant. This is justified in Fig. 3B, where the temporal evolution of the tumbling frequency after addition of $1 \mathrm{mM}$ of shikimate is shown. While the average tumbling frequency for the first 20 seconds corresponds to 0.13 tumbles/second, the minimum tumbling frequency within the same period of time corresponds to 0.04 tumbles/second. The origin of the discrepancy between these two values can be attributed to temporal processes involved in the diffusion of the chemical attractant in the culture's medium, as well as to temporal processes involved in cellular sensing and response to the chemical gradient. Note that the analysis was restricted to the initial 20 seconds of the video instead of its total duration (see blue rectangle in Fig. 3B). There are two reasons for this. The first one is related to the adaptation process that the cellular sensing machinery undergoes and the 
associated increase in the tumbling frequency. The second reason is related to the steady decrease over time in the number of cellular trajectories identified by TaxisPy, which compromises the representativeness of the tumbling frequencies calculated and potentially increases the noise at later time points. This observation was consistent over all recorded videos (see Fig. S1). For consistency, the tumbling frequency for the control condition was also calculated over a period of 20 seconds.

\subsection{Visualizing the adaptation process of the chemotactic machinery of $E$. coli.}

The two-component system controlling the chemotactic response of $E$. coli is able to adapt to external stimuli (Macnab \& Koshland 1972, Berg \& Tedesco 1975, Alon et al. 1999). Adaptation refers to the temporal process by which the cellular tumbling frequency returns to its pre-stimulation state. As a proof-of-concept and to demonstrate the utility and versatility of TaxisPy in elucidating this process, we stimulated an E. coli culture with $1 \mathrm{mM}$ L-aspartate and videotaped its swimming pattern at regular time intervals for 40 minutes. Each video was split into its constituting frames and these were analyzed by TaxisPy. Tumbling frequencies were calculated using time intervals of 10 seconds. These data are graphically represented by the blue dots in Fig. 4. Black dots in the same figure represent the tumbling frequency of a control culture without the addition of the chemical attractant.

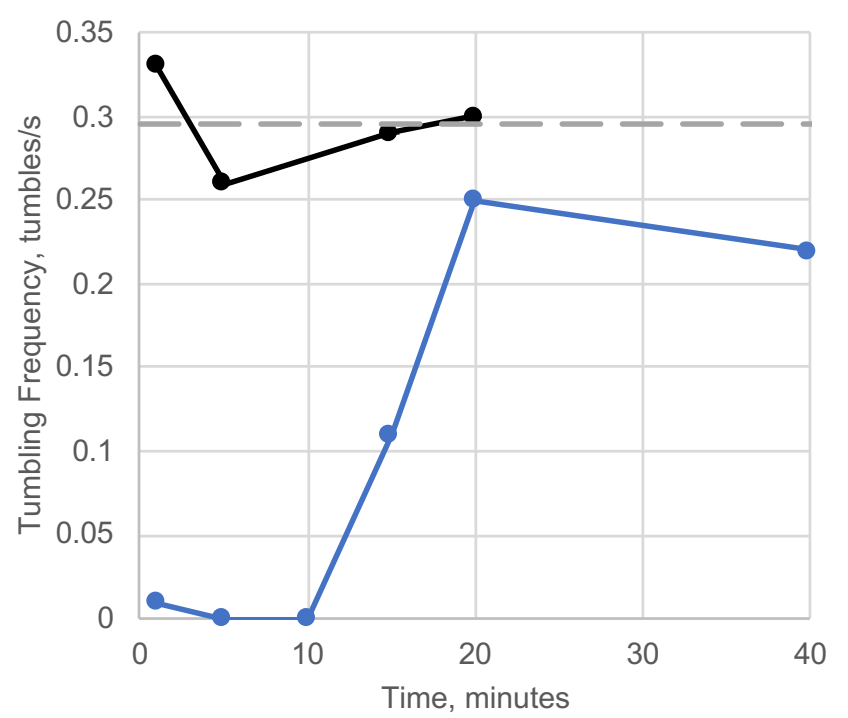

Figure 4. Adaptation of the chemotactic machinery of E. coli. L-aspartate (1 mM) was added to an $E$. coli culture at time 0 and its swimming pattern video tapped for 10 seconds in regular time intervals over 40 minutes. Each video was subsequently analyzed using TaxisPy to estimate the temporal evolution of the tumbling frequency. These data points are represented by the blue dots. The tumbling frequency of a control culture without attractant is represented by the black dots. The grey dashed line represents the 
average tumbling frequency of the unstimulated culture (0.295 tumbles/second). Each data point was generated from the analysis of 86 to 420 individual cellular trajectories.

The expected adaptation behavior is evident from the time course of the tumbling frequency for the stimulated culture (blue solid line in Fig. 4). As time goes by, the average tumbling frequency of the culture increases from values lower than 0.05 tumbles/second during the first 10 minutes, to reach a maximum value of 0.25 tumbles/second after 20 minutes. The adaptation process can be characterized by two parameters: the precision of adaptation and the adaptation time (Alon et al. 1999). The first parameter is defined as the ratio between the average tumbling frequency of the unstimulated culture and the average tumbling frequency of the stimulated culture after adaptation has been reached. The second parameter is defined as the time where the tumbling frequency of stimulated cells rises to halfway between its earliest measured value and its steady-state value. For the specific system under analysis, the adaptation time was 15 minutes and the precision of adaptation was 1.18 .

\section{Discussion}

A major difficulty related to the customized use of cell tracking software is the determination of key parameter values for the identification of tumbles or changes of direction. In the past, such parameters were empirically determined (Berg and Brown 1972, Harwood et al. 1989), which compromises the applicability of those parameters under different conditions - e.g., different microorganisms or stimuli. TaxisPy systematically addresses this issue by implementing various strategies designed to guide the selection of relevant parameter values. For instance, TaxisPy employs a genetic algorithm along with a condition-specific training set to identify optimal values for three key parameters: \# Frames, \# Smooth and Acc. Threshold. The first two parameters affect the way that TaxisPy smooth cellular velocity data by calculating the average from a number of frames (dictated by \# Frames) a certain number of times (determined by \# Smooth). Noisy positional data tend to require more smoothing cycles. TaxisPy analyzes the time course of the absolute acceleration -which is calculated from the smoothed velocity data- to identify tumbling events. We use the premise that a cell decreases its linear velocity when tumbling and increases it again thereafter. Thus, a change of direction or tumble is characterized by two adjacent peaks in the time course of the absolute acceleration. To avoid identifying normal fluctuations in the velocity during smooth swimming as a tumble, the acceleration peaks are required to surpass a threshold value dictated by Acc. Threshold. Since smoothing the velocity data decreases the height of the peaks in the acceleration plot, high 
values for \# Frames and \# Smooth are usually accompanied by low values for Acc. Threshold and vice versa.

One of the motivations for the development of TaxisPy was to offer a simple method for the quantitative analysis of bacterial chemotaxis. Following the way paved by previous studies that implemented video-based analysis of bacterial chemotaxis, we decided to base our method on the analysis of cellular motion data for the identification of tumbling frequencies. This led us to identify the necessity of condition-specific values for some parameters -\# Frames, \# Smooth and Acc. Threshold- and to develop features in TaxisPy for their identification. Even though constructing training sets and identifying optimal parameter values usually requires a couple of minutes, this procedure can become tedious if a large number of videos need to be processed.

A promising approach for the "parameter-free" identification of tumbles is the analysis of cellular trajectories by multilayer neural networks (Lecun et al. 1998, Ciregan et al. 2012). These networks could be first trained on a large data set of cellular trajectories -each one of them labeled with the number of tumbles- to then be used on new trajectories to estimate their number of tumbles. An advantage of such an approach would be that the model parameterization would be done just once and could be then applied for various microorganisms under different conditions. Paving the way towards this new approach, we are providing an initial training set in the Supplementary File 1, which was manually extracted and curated from the $P$. putida experiments and consists of over 700 cellular trajectories, each one provided with the number of observed tumbles.

In this study, we used two different experimental set-ups to study the bacterial chemotactic response. For $P$. putida, the temporal response to shikimate pulses of varying concentration were measured on microscope glass slides. Using the minimum tumbling frequency during the first 20 seconds of each experiment, we were able to quantitatively capture

417 the effect of the shikimate concentration on the swimming behavior. As expected, we observed

418 a decrease in the tumbling frequency as the concentration of shikimate was increased (Fig. 3A).

419 Increasing the shikimate concentration over $100 \mu \mathrm{M}$ did not seem to have a further effect on the 420 chemotactic response of $P$. putida. Additionally, values calculated for the tumbling frequency

421 were within the same order of magnitude as previous reports (Harwood et. al. 1989), which

422 validates the computations performed by TaxisPy.

423 As mentioned in the results section and shown in Figs. 3B and S1, the number of 424 trajectories identified by TaxisPy consistently decreased during the course of each experiment.

425 This is potentially related to a temperature or light-dependent taxis away from the observation

426 field of the microscope. An indirect support to this claim is provided by a report by Paster and 
427 Ryu (2008), in which a tactic response was observed in E. coli as a function of temperature

428 gradients. The authors showed that at temperatures below $31^{\circ} \mathrm{C}$, the response to thermal

429 stimuli is similar to the chemotactic response. However, at temperatures above $31^{\circ} \mathrm{C}$, some

430 cells showed an inverted response, switching from warm- to cold-seeking behavior. Additional

431 factors to be considered might be related with oxygen depletion and the accompanied overall

432 loss of bacterial motility (Douarche et al. 2009) and with cellular migration towards the nearest

433 air/water interface due to aerotaxis (Taylor 1983) and away from the focus of the microscope.

434 From a practical point of view, a steady decrease in the number of cellular trajectories over time

435 has two implications. First, it suggests that the uncertainty of data extracted from adaptation

436 experiments performed on glass slides will increase over time, since the number of trajectories

437 used to calculate tumbling frequencies will continuously decrease. Second, it poses a biological

438 constraint on the maximal length of the videos when the swimming pattern is observed on a

439 glass slide. In line with these observations, we limited the analysis of each P. putida video to the

440 first 20 seconds.

441 In the case of E. coli, a different experimental set-up was followed. Since the adaptation

442 time exhibited by this microorganism under the studied conditions is in the order of magnitude of

443 minutes, the actual adaptation process was conducted in an Eppendorf tube instead of in a

444 microscope slide. Samples were taken from the vessel, which was kept under shaking

445 conditions, and analyzed under the microscope for 10 seconds. In this way, we were able to

446 estimate both the adaptation time and the precision of adaptation of the culture. The estimated

447 value for the adaptation time of 15 minutes and the precision of adaptation of 1.18 are

448 consistent with previously reported values (Alon et. al. 1999). These results serve as further

449 validation of our methods.

450 TaxisPy was designed for users with limited programming knowledge. Its user interface

451 provides necessary tools to split a video into its constituting frames, identify cells in individual

452 frames, link these to obtain trajectories, filter anomalous trajectories and calculate tumbling

453 frequencies. Thanks to its convenient distribution through Docker, intuitive user interface and

454 biologically feasible results, TaxisPy represents a valuable computational tool for the

455 quantitative analysis of bacterial chemotaxis. 


\section{Acknowledgements}

462

463 This work was supported by grants from the National Science Foundation to R.E.P and

464 M.A.S (MCB 1716833), and the USDA National Institute of Food and Agriculture,

$465 \quad$ Hatch/Evans-Allen/Mclntire Stennis project 1020219 to R.E.P.

466

467

6. Author Contributions

468

469

Conceptualization, R.E.P.; Methodology, R.A.S. and M.A.V.; Software, M.A.V; Validation, R.A.S and M.A.V.; Writing, M.A.V., R.A.S. and R.E.P; Supervision, R.E.P. and M.A.S., Funding Acquisition, R.E.P and M.A.S.

472

473

474

7. Declaration of Interests

475

The authors declare no conflict of interests 


\section{References}

1. Adler, J. (1969). Chemoreceptors in bacteria. Science 166:1588-1597

2. Adler, J. (1973). A method for measuring chemotaxis and use of the method to determine optimum conditions for chemotaxis by Escherichia coli. J. Gen. Microbiol. 74(1):77-91

3. Allan, D., van der Wel, C., Keim, N., Caswell, T.A., Wieker, D., Verweij, R., Reid, C., Grueter, L., Ramos, K., Perry, R.W., Boulogne, F., Sinha, P., Bruot, N., Uieda, L., Katis, J., Mary, H., Ahmadia, A. (2019) Trackpy v0.4.2. Zenodo. https://doi.org/10.5281/zenodo.3492186

4. Alon, U., Camarena, L., Surette, M.G., Aguera y Arcas, B., Liu, Y., Leibler, S., and Stock, J.B. (1998). Response regulator output in bacterial chemotaxis. EMBO J. 17(15): 4238-4248

5. Alon, U., Surette, M. G., Barkai, N., and Leibler, S. (1999). Robustness in bacterial chemotaxis. Nature. 397(6715):168-71

6. Amsler CD (1996). Use of computer-assisted motion analysis for quantitative measurements of swimming behavior in peritrichously flagellated bacteria. Anal Biochem. 1;235(1):20-5

7. Berg, H.C., and Brown, D.A (1972). Chemotaxis in Escherichia coli analysed by Threedimensional Tracking. Nature 239:500-4

8. Berg, H.C. and Tedesco, P. (1975). Transient response to chemotaxis stimuli in Escherichia coli. Proc. Natl. Acad. Sci. USA 72, 3235-3239

9. Ciregan, D., Meier, U. and Schmidhuber, J. (2012). Multi-column deep neural networks for image classification. IEEE Conference on Computer Vision and Pattern Recognition, Providence, RI, 2012, pp. 3642-3649.

doi: 10.1109/CVPR.2012.6248110

10. Crocker, J. C., and Grier, D. G. (1996). Methods of Digital Video Microscopy for Colloidal Studies. J. Colloid Interf. Sci., 179(1), 298-310. http://doi.org/10.1006/jcis.1996.0217

11. Ditty J.L. and Parales R.E. (2015) Protocols for the Measurement of Bacterial Chemotaxis to Hydrocarbons. In: McGenity T., Timmis K., Nogales B. (eds) Hydrocarbon and Lipid Microbiology Protocols. Springer Protocols Handbooks. Springer, Berlin, Heidelberg

12. Douarche, C., Buguin, A., Salman, H., Libchaber, A. (2009) E. coli and oxygen: a motility transition. Phys Rev Lett. 15;102(19):198101

13. Fortin, F.A., De Rainville, F.M., Gardner, M.A, Parizeau, M., Gagné, C. (2012) DEAP: Evolutionary Algorithms Made Easy. Journal of Machine Learning Research. 13, 21712175 
28. Sambrook, J., Fritch, E.F., Maniatis, T. (1989) Molecular cloning: a laboratory manual, 2nd edn. Cold Spring Harbor Laboratory, Cold Spring Harbor

29. Stanier, R.Y., Palleroni, N.J., Doudoroff, M. (1966). The aerobic pseudomonads: a taxonomic study. J Gen Microbiol 43, 159-271 10.1099/00221287-43-2-159

30. Staropoli, J.F., Alon, U. (2000). Computerized Analysis of Chemotaxis at Different Stages of Bacterial Growth. Biophys. J. 78:513-519

31. Taylor, B.L. (1983) How do bacteria find the optimal concentration of oxygen? Trends Biochem Sci. 8(12): 438-441.

32. Tso, WW., and Adler, J. (1974). Negative chemotaxis in Escherichia coli. J Bacteriol. 118(2):560-76

33. Webre, D.J, Wolanin P.M., and Stock, J.B. (2003). Bacterial chemotaxis. Curr Biol. 13(2):R47-9. DOI: 10.1016/s0960-9822(02)01424-0 